\title{
Using structural neuroanatomy to identify trauma survivors with and without post-traumatic stress disorder at the individual level
}

\author{
Q. Gong ${ }^{1 *} \uparrow$, L. $\mathrm{Li}^{2} \dagger$, S. Tognin ${ }^{3}$, Q. Wu ${ }^{1}$, W. Pettersson-Yeo ${ }^{3}$, S. Lui ${ }^{1}$, X. Huang ${ }^{1}$, A. F. Marquand ${ }^{4}$ \\ and A. Mechelli ${ }^{3}$ \\ ${ }^{1}$ Huaxi MR Research Center (HMRRC), Department of Radiology, State Key Laboratory of Biotherapy, West China Hospital of Sichuan University, \\ Chengdu, Sichuan, People's Republic of China \\ ${ }^{2}$ Mental Health Institute, The Second Xiangya Hospital of Central South University, Changsha, Hunan, People's Republic of China \\ ${ }^{3}$ Department of Psychosis Studies, Institute of Psychiatry, King's College London, London, UK \\ ${ }^{4}$ Department of Clinical Neuroscience, Centre for Neuroimaging Sciences, Institute of Psychiatry, King's College London, London, UK
}

Background. At present there are no objective, biological markers that can be used to reliably identify individuals with post-traumatic stress disorder (PTSD). This study assessed the diagnostic potential of structural magnetic resonance imaging (sMRI) for identifying trauma-exposed individuals with and without PTSD.

\begin{abstract}
Method. sMRI scans were acquired from 50 survivors of the Sichuan earthquake of 2008 who had developed PTSD, 50 survivors who had not developed PTSD and 40 healthy controls who had not been exposed to the earthquake. Support vector machine (SVM), a multivariate pattern recognition technique, was used to develop an algorithm that distinguished between the three groups at an individual level. The accuracy of the algorithm and its statistical significance were estimated using leave-one-out cross-validation and permutation testing.

Results. When survivors with PTSD were compared against healthy controls, both grey and white matter allowed discrimination with an accuracy of $91 \%(p<0.001)$. When survivors without PTSD were compared against healthy controls, the two groups could be discriminated with accuracies of $76 \%(p<0.001)$ and $85 \%(p<0.001)$ based on grey and white matter, respectively. Finally, when survivors with and without PTSD were compared directly, grey matter allowed discrimination with an accuracy of $67 \%(p<0.001)$; in contrast the two groups could not be distinguished based on white matter.

Conclusions. These results reveal patterns of neuroanatomical alterations that could be used to inform the identification of trauma survivors with and without PTSD at the individual level, and provide preliminary support to the development of SVM as a clinically useful diagnostic aid.
\end{abstract}

Received 28 June 2012; Revised 15 February 2013; Accepted 15 February 2013; First published online 3 April 2013

Key words: Magnetic resonance imaging, natural disaster, post-traumatic stress disorder (PTSD), support vector machine (SVM), voxel-based morphometry (VBM).

\section{Introduction}

Post-traumatic stress disorder (PTSD) is an anxiety disorder that may develop in individuals who have experienced or witnessed severe traumatic events and is characterized by persistent re-experience of the traumatic event, avoidance of trauma-related stimuli and hypervigilance (Yehuda \& Flory, 2007). Individuals with PTSD are known to have high rates of co-morbidity with other Axis I psychiatric disorders, somatic

\footnotetext{
* Address for correspondence: Q. Gong, M.D., Ph.D., Huaxi Magnetic Resonance Research Center (HMRRC), Department of Radiology, West China Hospital of Sichuan University, Chengdu, 610041, People's Republic of China.

(Email: qiyonggong@hmrrc.org.cn)

$\dagger$ These authors contributed equally to this work.
}

disorders and mild cognitive impairment (Lanius et al. 2007; Pace \& Heim, 2011). Therefore, it can be difficult to discern the symptoms and obtain an accurate diagnosis. In addition, cases of pseudo-PTSD, in which a patient's presentation is but a simulation of the actual syndrome, can be particularly difficult to identify due to the traditional assumption that a patient's reported symptoms can be accepted as valid (Rosen \& Taylor, 2007; Sartori et al. 2011). A number of neuroimaging studies have therefore compared individuals who did and did not develop PTSD following exposure to a traumatic event, in order to identify possible diagnostic biomarkers (Shin et al. 2006; Nardo et al. 2010; Robinson \& Shergill, 2011). The results have revealed volumetric reductions in those who had become ill, relative to those who had not, within a distributed fronto-limbic network which 
includes the dorsolateral and ventromedial prefrontal cortices, amygdala and hippocampus (Shin et al. 2006; Nardo et al. 2010; Robinson \& Shergill, 2011). However, the neuroimaging studies published so far have used standard mass-univariate analytical methods and therefore have only been able to report differences at the group level, which is of little use in clinical practice where doctors need to make clinical decisions about individuals. At present, there are no reliable biomarkers that can be used to identify trauma-exposed individuals with and without PTSD, and the diagnosis of this disorder is still entirely reliant on clinical presentation and psychometric measures (Regier et al. 2009).

In order to enhance the translational impact of the results, there has been a recent shift toward the use of multivariate pattern recognition techniques, such as support vector machine (SVM). This technique has two main advantages compared with standard massunivariate analytical methods that are typically used in neuroimaging (Lao et al. 2004; Norman et al. 2006; Brammer, 2009; Pereira et al. 2009; Orrù et al. 2012). First, it allows inferences to be made at the level of the individual and therefore yields results with high translational potential in everyday clinical practice. Second, it takes inter-regional correlations into account and therefore is sensitive to differences that are subtle and spatially distributed; as such, it provides an ideal framework for investigating psychiatric disorders that affect a distributed network of regions. More specifically, SVM is a technique for classifying individual observations into distinct groups or classes based on high-dimensional data and consists of a training phase and a testing phase (Vapnik, 1995). In the training phase, a 'decision function' or 'hyperplane' is developed which best distinguishes between the two experimental groups of interest (e.g. patients versus controls). In the testing phase, this decision function is used to predict the group to which a new observation belongs. The overall accuracy of the algorithm depends on its sensitivity (i.e. the proportion of patients identified as having the disorder) and specificity (i.e. the proportion of controls identified as not having the disorder), and provides an estimate of how well it can be expected to generalize to future cases.

In recent years, a growing number of studies have applied SVM to neuroimaging data to examine a range of psychiatric and neurological conditions including schizophrenia, depression, Alzheimer's disease and autistic spectrum disorder (e.g. Davatzikos et al. 2005; Fan et al. 2008a; Fu et al. 2008; Klöppel et al. 2008; Costafreda et al. 2009; Koutsouleris et al. 2009; Ecker et al. 2010; Gong et al. 2011). The results of these studies have been promising, indicating good diagnostic and prognostic accuracies and a high level of generalizability across research centres. However, none of the studies published so far has used SVM to examine neuroanatomical alterations in PTSD; thus, it remains unclear whether those with PTSD can be accurately identified at the level of the individual.

The aim of the current investigation was therefore to assess the diagnostic potential of structural magnetic resonance imaging (MRI) for identifying traumaexposed individuals with and without PTSD. We acquired structural MRI data from a total of 150 volunteers including 50 survivors of the Sichuan earthquake of 2008 who had developed PTSD, 50 survivors who had not developed PTSD and 40 healthy controls who had not been exposed to the earthquake. We then analysed the data using SVM in order to make inferences at the level of the individual. Based on animal models suggesting that exposure to a brief period of intense stress is sufficient to cause significant neuroanatomical changes (Holmes \& Wellman, 2009) and recent evidence that stressful life events lead to longitudinal changes in the human brain (Papagni et al. 2011), we expected that both groups of survivors could be discriminated from healthy controls with statistically significant accuracy. Furthermore, based on the results of previous neuroimaging studies of PTSD (Shin et al. 2006; Nardo et al. 2010; Robinson \& Shergill, 2011), we hypothesized that the direct comparison between trauma-exposed individuals with and without PTSD would allow accurate discrimination between the two groups at an individual level.

\section{Methods}

\section{Sample}

At 14.28 hours on 12 May 2008, an earthquake measuring 8.0-magnitude devastated the Sichuan Province of China. We recruited 100 survivors of the earthquake including 50 who fulfilled the diagnostic criteria for PTSD and 50 who did not meet such criteria using the PTSD Checklist (Weathers et al. 1994), a 17-item self-report measure of symptoms of PTSD, and the Clinician-Administered PTSD Scale (Blake et al. 1995), a 30-item structured interview based on the DSM-IV criteria for PTSD. Inclusion criteria for the two groups of survivors included: (i) physically experiencing the earthquake; (ii) personally witnessing death, serious injury or the collapse of buildings; and (iii) not suffering any physical injury. Exclusion criteria included psychiatric co-morbidity assessed using the Structured Clinical Interview for DSM-IV (First et al. 1995), a history of psychiatric or neurological disorders, recent medication that might affect brain function, alcohol or drug abuse and pregnancy. The acquisition of neuroimaging and clinical data from survivors took place 
Table 1. Demographic and clinical characteristics of participants

\begin{tabular}{llll}
\hline & $\begin{array}{l}\text { Survivors } \\
\text { with } \\
\text { PTSD }\end{array}$ & $\begin{array}{l}\text { Survivors } \\
\text { without } \\
\text { PTSD }\end{array}$ & $\begin{array}{l}\text { Healthy } \\
\text { controls }\end{array}$ \\
\hline $\begin{array}{l}\text { Subjects, } n \\
\text { Mean age, }\end{array}$ & 50 & 50 & 50 \\
years (S.D.) & $42.58(10.22)$ & $43.28(10.14)$ & $42.43(11.51)$ \\
$\begin{array}{c}\text { Gender, } n \\
\text { Male }\end{array}$ & 18 & & \\
$\begin{array}{l}\text { Female } \\
\text { Mean PCL }\end{array}$ & 32 & 18 & 18 \\
(s.D.) & $46.54(11.78)$ & $29.00(7.72)$ & 32 \\
$\begin{array}{c}\text { Mean CAPS } \\
\text { (S.D.) }\end{array}$ & $53.88(16.60)$ & $23.63(12.38)$ & \\
\hline
\end{tabular}

PTSD, Post-traumatic stress disorder; S.D., standard deviation; PCL, PTSD Checklist; CAPS, ClinicianAdministered PTSD Scale.

between 263 and 468 days after the earthquake, with an average interval of 384.33 (s.D. $=58.43$ ) days. In addition, we used data from 40 healthy participants recruited by advertisement from the same region and scanned with the same protocol shortly before the earthquake. Exclusion criteria included history of psychiatric or neurological disorders, recent medication that might affect brain function, alcohol or drug abuse and pregnancy. The three groups were carefully matched for age and gender by pairing each subject in one group with another subject of the same gender and the same age ( \pm 3 years) in the other two groups (Table 1). Inspection of neuroanatomical scans (i.e. T1- and T2-weighted images) by an experienced neuroradiologist did not reveal gross abnormalities in any of the participants. Informed written consent was obtained from all participants and the research protocol was approved by the local ethics committee.

\section{MRI acquisition}

All participants were scanned using a 3T MRI system (EXCITE, General Electric, USA) with a volumetric three-dimensional spoiled gradient-recalled sequence (repetition time $=8.5 \mathrm{~ms}$, echo time $=3.4 \mathrm{~ms}$, flip angle $=12^{\circ}$, slice thickness $=1.0 \mathrm{~mm}$ ) with an eight-channel phase array head coil. A field of view of $24 \mathrm{~cm}^{2}$ was used with an acquisition matrix comprising 256 readings of 128 phase-encoding steps, producing 156 contiguous coronal slices with slice thickness of $1.0 \mathrm{~mm}$ and in-plane resolution of $0.47 \times 0.47 \mathrm{~mm}$.

\section{Pre-processing}

After checking for scanner artifacts and gross anatomical abnormalities for each subject, we reoriented the original images along the anterior-posterior commissure line and set the anterior commissure as the origin of the spatial coordinates to assist the normalization algorithm. The new segmentation procedure implemented in SPM8 (http://www.fil.ion.ucl.ac.uk/ spm), running under Matlab 7.1 (Math Works, USA) was used to segment all the images into grey matter (GM) and white matter (WM), i.e. maps of probability values representing the probability of a voxel containing a specific tissue type. A fast diffeomorphic image registration algorithm (Ashburner, 2007), available as a toolbox in SPM8, was used to warp the GM and WM partitions into a new study-specific reference space representing an average of all the subjects included in the analysis. As an initial step, a set of studyspecific templates and the corresponding deformation fields, required to warp the data from each subject to the new reference space, were created using the GM and WM partitions. Each subject-specific deformation field was used to warp the corresponding GM and WM partitions into the new reference space with the aim of maximizing accuracy and sensitivity (Yassa \& Stark, 2009); finally, the warped GM and WM partitions were separately affine transformed into the Montreal Neurological Institute (MNI) space and smoothed with a standard $8 \mathrm{~mm}$ full-width at half-maximum Gaussian kernel. An additional 'modulation' step was used to scale the GM and WM probability values by the Jacobian determinants of the deformations to ensure that the total amount of GM and WM in each voxel was conserved after the registration. The resulting GM and WM partitions had an isotropic resolution of $1.5 \mathrm{~mm}$ and included a total of 275042 and 169491 voxels, respectively.

\section{$S V M$}

After pre-processing, we used SVM (Burges, 1998) as implemented in the PROBID software package (http:// www.brainmap.co.uk/probid.htm) to investigate the potential of whole-brain structural MRI images for discriminating amongst the three experimental groups. Individual brain scans were treated as points located in a high-dimensional space defined by the GM or WM values in the pre-processed images. A linear decision boundary in this high-dimensional space was defined by a 'hyperplane' that separated the individual brain scans according to a class label (e.g. survivors with versus without PTSD). The optimal hyperplane was computed based on the whole multivariate pattern of GM values across each structural MRI image. A linear rather than a non-linear kernel SVM was used in order to reduce the risk of overfitting the data and to allow direct extraction of the weight vector as an image (i.e. the SVM discrimination 
map). The PROBID software allows a linear kernel matrix (measuring similarity between all pairs of brain images) to be pre-computed and supplied to the classifier. This approach affords a substantial increase in computational efficiency and permits whole-brain classification without requiring explicit dimensionality reduction. A 'leave-one-out' crossvalidation method (Hastie \& Tibshirani, 2001) was used which involved excluding a single subject from each group and training the classifier using the remaining subjects; the subject pair excluded was then used to test the ability of the classifier to reliably distinguish between categories (e.g. survivors with versus without PTSD). This procedure was repeated for each subject pair in order to assess the overall accuracy of the SVM. Statistical significance of the overall classification accuracy was determined by permutation testing; this involved repeating the classification procedure 1000 times with a different random permutation of the training group labels and counting the number of permutations achieving higher sensitivity and specificity than the true labels. We repeated this procedure for GM and WM images separately in order to assess the predictive power of each tissue type.

\section{Mass-univariate analysis}

In order to compare the results of our multivariate approach with the standard mass-univariate analysis using the general linear model, a total of three paired $t$ tests comparing those subjects used for the corresponding SVM comparisons were conducted for GM and $\mathrm{WM}$, respectively. These $t$ tests were implemented in SPM8 software (http://www.fil.ion.ucl.ac.uk/spm) running under Matlab 7.1 (Math Works, USA). Statistical inferences were made at $p<0.05$ after familywise error (FWE) correction for multiple comparisons. Regions reported in Tables S1-S9 of the Supplementary material were identified using the Automated Anatomical Labeling atlas as implemented in PickAtlas software (http://fmri.wfubmc.edu/software/ PickAtlas).

\section{Results}

\section{Demographics and clinical scores}

The three groups were perfectly matched for gender and did not differ with respect to age $(F=0.143, p=$ 0.867). However, the interval between the earthquake and scanning differed between survivors with (355.64 days, S.D. $=66.97)$ and without (413.02 days, S.D. $=$ 27.06) PTSD $[t=-5.62$, degrees of freedom $(\mathrm{df})=98$, $p<0.001]$. As expected, survivors with PTSD scored higher than survivors without PTSD both on the PTSD Checklist $(t=8.80, \mathrm{df}=98, p<0.001)$ and on the
Clinician-Administered PTSD Scale $(t=9.94, \mathrm{df}=98$, $p<0.001$ ) (Table 1).

\section{Survivors with PTSD versus healthy controls}

We first compared survivors who had developed PTSD against healthy controls. The application of SVM to GM images allowed discrimination between the two groups with a sensitivity of $92 \%$ and a specificity of $90 \%$, resulting in an accuracy of $91 \%$; permutation testing indicated that such accuracy was statistically significant $[p<0.001$; see Fig. S1 in the Supplementary material for receiver operating characteristic (ROC) curve]. The application of SVM to WM images yielded a similar pattern of results, namely a sensitivity of $97 \%$, a specificity of $85 \%$ and an accuracy of $91 \%(p<0.001$; see Fig. S1 in the Supplementary material for ROC curve). When GM and WM were considered simultaneously, sensitivity, specificity and accuracy were $95,87.5$ and $91.25 \%$, respectively $(p<$ 0.001). The use of an arbitrary threshold corresponding to the top $30 \%$ of the maximum absolute weight vector score revealed that discrimination between the two groups was driven by GM and WM differences in a widely distributed network of prefrontal, temporal, parietal and occipital regions (Fig. 1; see Tables S1 and $S 2$ in the Supplementary material for a full list).

In addition to the multivariate analysis, we performed a standard mass-univariate analysis for comparison. This revealed that survivors with PTSD showed reduced GM and WM relative to healthy controls within a highly distributed bilateral network which included prefrontal, temporal and parietal regions, the cerebellum and the putamen $(p<0.05$ after FWE correction). These GM and WM differences are reported in detail in Tables S3 and S4 of the Supplementary material.

\section{Survivors without PTSD versus healthy controls}

We then compared survivors who had not developed PTSD against healthy controls. Using GM images, SVM allowed discrimination between the two groups with a sensitivity of $77 \%$ and a specificity of $75 \%$, resulting in an accuracy of $76 \%(p<0.001$; see Fig. S2 in the Supplementary material for ROC curve). A similar pattern of results was found using WM images, namely a sensitivity of $87 \%$, a specificity of $82 \%$ and an accuracy of $85 \% \quad(p<0.001$; see Fig. S2 in the Supplementary material for ROC curve). When GM and WM were considered simultaneously, sensitivity, specificity and accuracy were 87.5, 80 and $83.75 \%$, respectively $(p<0.001)$. The use of an arbitrary threshold corresponding to the top $30 \%$ of the maximum absolute weight vector score showed that discrimination between the two groups was driven by GM 


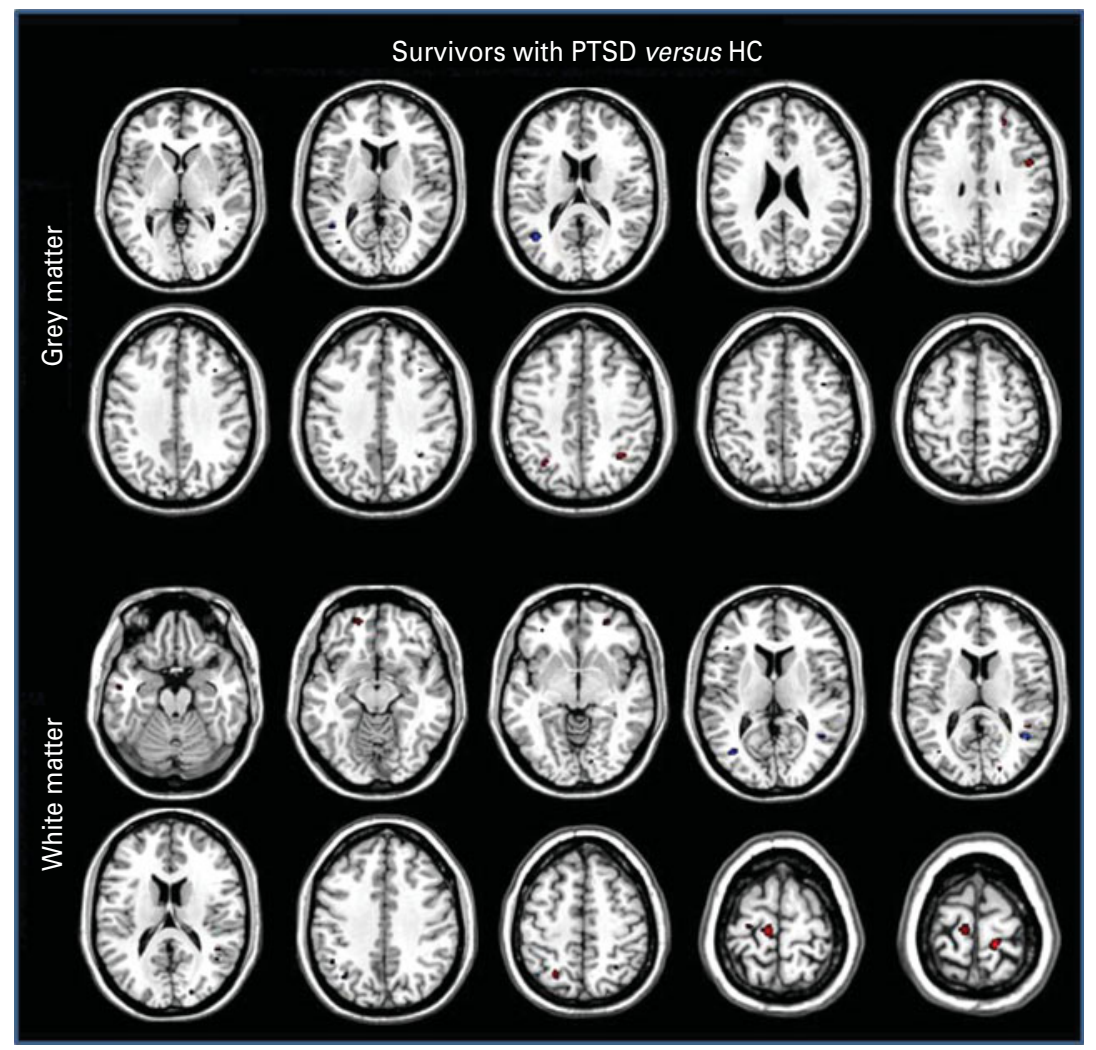

Fig. 1. Grey and white matter regions that showed the highest discriminative value for the comparison between survivors with post-traumatic stress disorder (PTSD) and healthy controls (HC). Regions were identified by setting the threshold to the top $30 \%$ of the maximum absolute weight vector score. Red indicates higher values in the group of survivors with PTSD, while blue indicates higher values for the HC group.

and WM differences in several prefrontal, temporal, parietal and occipital regions (Fig. 2; see Tables S5 and $\mathrm{S} 6$ in the Supplementary material for a full list).

The standard mass-univariate analysis revealed that survivors without PTSD relative to the healthy controls had reduced GM in the bilateral middle temporal gyrus, left parahippocampal gyrus, left lentiform gyrus, right inferior frontal gyrus, left cerebellum, left cuneus and right putamen $(p<0.05$ after FWE correction); the only GM region to show increased volume in survivors without PTSD relative to the healthy controls was the left putamen whereas there were no WM regions that showed the same effect. These differences are reported in detail in Tables S7 and S8 of the Supplementary material.

\section{Survivors with PTSD versus survivors without PTSD}

Finally we directly compared survivors who had developed PTSD against survivors who had not. Using GM images, SVM allowed discrimination between the two groups with a sensitivity of $66 \%$, a specificity of $68 \%$ and an accuracy of $67 \%(p<0.001$; see Fig. S3 in the Supplementary material for ROC curve). The use of an arbitrary threshold corresponding to the top 30\% of the maximum absolute weight vector score revealed that this discrimination was driven by GM differences in a highly distributed network that included prefrontal, temporal, parietal and occipital regions as well as subcortical structures (Fig. 3; see Table S9 in the Supplementary material for a full list). In contrast the two groups could not be distinguished at an individual level using WM images (sensitivity $58 \%$, specificity $58 \%$, accuracy $58 \%, p>0.05$ ). When GM and WM were considered simultaneously, sensitivity, specificity and accuracy were 76,50 and $62 \%$, respectively $(p=0.006)$. The standard mass-univariate analysis did not detect any significant differences between survivors with and without PTSD after correction for multiple comparisons, in either GM or WM.

\section{Network of interest analyses}

For completeness, we repeated the above analyses using an a priori mask comprising regions implicated in PTSD, i.e. the bilateral prefrontal cortex, amygdala and hippocampus (see Fig. S4 in the Supplementary material) (Shin et al. 2006; Nardo et al. 2010; Robinson \& Shergill, 2011). For most comparisons 


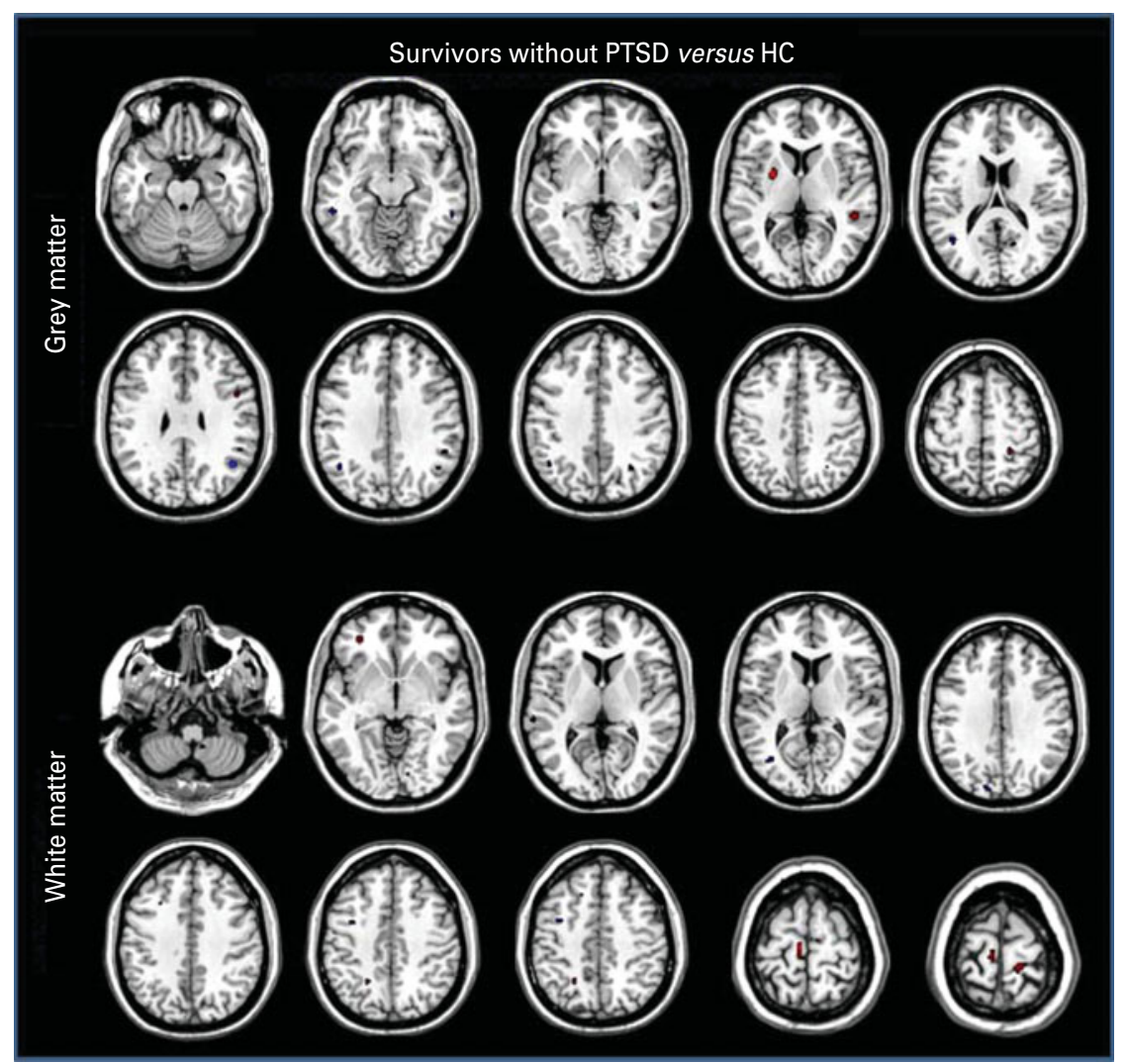

Fig. 2. Grey and white matter regions that showed the highest discriminative value for the comparison between survivors without post-traumatic stress disorder (PTSD) and healthy controls (HC). Regions were identified by setting the threshold to the top $30 \%$ of the maximum absolute weight vector score. Red indicates higher values in the group of survivors without PTSD, while blue indicates higher values for the HC group.

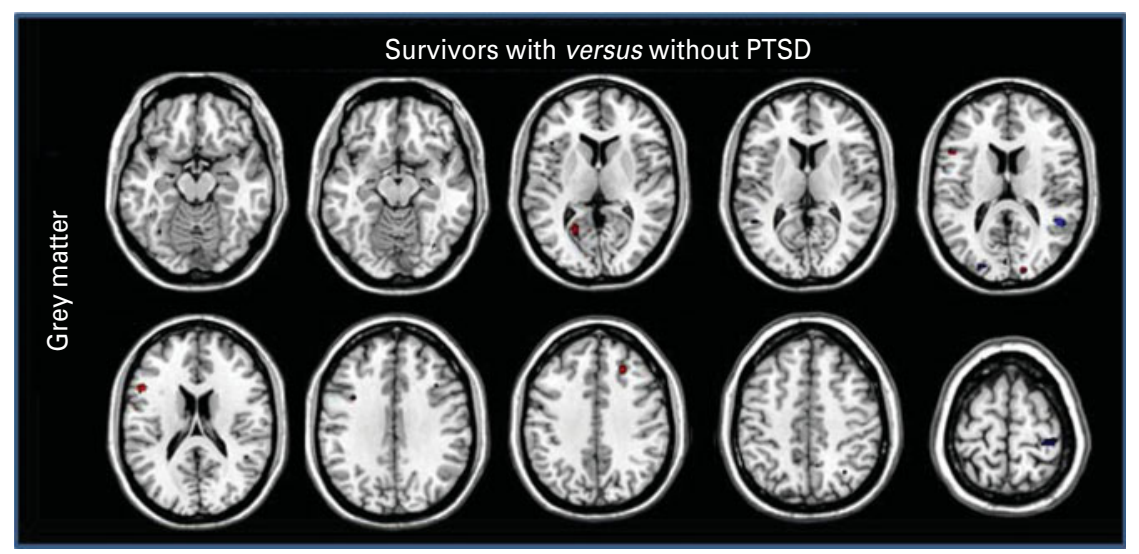

Fig. 3. Grey matter regions that showed the highest discriminative value for the comparison between survivors with and without post-traumatic stress disorder (PTSD). Regions were identified by setting the threshold to the top $30 \%$ of the maximum absolute weight vector score. Red indicates higher values in the group of survivors with PTSD, while blue indicates higher values for survivors without PTSD.

accuracy was still statistically significant but lower compared with the whole-brain analyses (see Table S10 in the Supplementary material), suggesting that discrimination was informed by regions outside the bilateral fronto-limbic network that is traditionally associated with PTSD.

\section{Discussion}

The results demonstrate that multivariate analysis of structural MRI allows discrimination between trauma-exposed individuals and healthy controls with high levels of accuracy (76-91\%, $p<0.001)$; this 
finding was replicated using GM and WM, indicating that neuroanatomical differences between the two groups were expressed in both tissue types. In addition, the results show that it is possible to discriminate between trauma-exposed individuals with and without PTSD at an individual level with above-chance accuracy $(67 \%, p<0.001)$; such discrimination was obtained using GM but not WM, suggesting that neuroanatomical differences between the two groups were tissue-specific. This pattern of results provides preliminary support to the development of SVM as a clinically useful diagnostic tool in PTSD. Such a tool could potentially be used in a clinical setting to inform the clinical evaluation of those difficult to categorize using traditional methods of clinical assessment alone (Lanius et al. 2007; Pace \& Heim, 2011). In addition, it could potentially be used in a forensic setting as an objective means of reducing controversy in evaluations of mental illness and minimizing errors in detecting malingering (Rosen \& Taylor, 2007; Sartori et al. 2011). In contrast, no significant regions were detected for the comparison between survivors with and without PTSD using standard mass-univariate techniques in which each voxel is considered as a spatially independent unit. This provides support to the idea that multivariate methods such as SVM may be better suited to the development of a real-world clinical diagnostic tool than standard mass-univariate techniques (Brammer, 2009).

Successful discrimination between the two groups of survivors was driven by neuroanatomical alterations in a widespread network of prefrontal, temporal, parietal and occipital regions as well as subcortical structures (Fig. 3). Furthermore, the use of a fronto-limbic mask resulted in generally lower accuracy relative to the whole-brain analyses, indicating that regions outside the bilateral fronto-limbic network that is traditionally associated with PTSD contributed to discrimination. In multivariate methods such as SVM, an individual region may display high discriminative power due to two possible reasons: (i) a difference in volume between groups in that region; and (ii) a difference in the correlation between that region and other areas between groups. Thus, the widespread network identified in the present investigation should be interpreted as a spatially distributed pattern rather than as individual regions. In addition, it should be noted that this widespread network was identified using an arbitrary threshold and that the discrimination was in fact informed by all voxels in the brain since no feature extraction was employed. Thus, one should be cautious when drawing inferences regarding specific regions out of the context of the overall pattern using multivariate methods.
In the present investigation, we could not establish whether the neuroanatomical differences that led to accurate discrimination between survivors with and without PTSD reflected neuroplastic changes that occurred after the earthquake or pre-existing differences associated with individual variability in psychological vulnerability. However, the fact that the two groups of survivors could be discriminated from healthy controls with high levels of accuracy suggests that our result may reflect neuroplastic changes after the earthquake rather than pre-existing differences. This interpretation is supported by animal models suggesting that exposure to a brief period of intense stress is sufficient to cause significant neuroanatomical changes (Holmes \& Wellman, 2009) and recent evidence that stressful life events lead to longitudinal changes in the human brain (Papagni et al. 2011). We also note that on average survivors who met criteria for PTSD were more likely to have been scanned sooner after the earthquake than those who did not. This points to time from the earthquake as a potential critical variable modulating the differences in clinical symptomatology and structural neuroanatomy between the two groups, and is therefore consistent with the interpretation of our results in terms of neuroplastic changes.

The present study has a number of methodological limitations. First, all healthy participants were scanned shortly before the earthquake and therefore some of the differences between this group and survivors could be explained by changes in image quality before and after the earthquake; however, careful examination of phantom images acquired before and after the earthquake found no evidence for such changes and furthermore this limitation is not relevant to the comparison between survivors who did and did not develop PTSD. Second, all data were acquired using the same scanner and acquisition sequence and therefore we were unable to make inference regarding the generalizability of any of the successful classifiers across different research centres. Nevertheless, a recent investigation of MRI changes in Alzheimer dementia provides preliminary evidence that the methodology can be generalized across different research centres (Klöppel et al. 2008). Third, the three subject groups were compared using a binary rather than a multi-class classification approach, which would have provided a closer approximation of how differential diagnostic decisions are made in real-world clinical practice. However, since multiple binary classifiers for each possible comparison were generated in the current study, a quantitative estimate is still provided demonstrating the relative ease, or difficulty, with which subjects from each group may be differentiated from the subjects of every other group, with respect to each 
data type. Finally, the development of a diagnostic algorithm in SVM is based on the distinction between patients and controls in the training data, which in turn relies on traditional clinical assessment. It follows that the application of SVM to neuroimaging data can only reach the same level of diagnostic accuracy as traditional methods of clinical assessment. Nevertheless, such application could still be useful as an objective means of reducing controversy in forensic evaluations of mental insanity and minimizing errors in detecting malingering (Rosen \& Taylor, 2007; Sartori et al. 2011). For a more comprehensive discussion of the limitations of the use of SVM in psychiatry and neurology, see our recent review (Orrù et al. 2012).

In conclusion, we have reported that multivariate analysis to MRI allows the identification of traumaexposed individuals with high accuracy, and furthermore identifies trauma-exposed individuals who have and have not developed PTSD with statistically significant accuracy. These results reveal patterns of neuroanatomical alterations that could be used to inform the identification of trauma survivors with and without PTSD at the individual level, and provide preliminary support to the development of SVM as a clinically useful diagnostic aid. Nevertheless, it should be stressed that the eventual use of this technique in the real world would ultimately require a greater level of accuracy than that found in the present study. Greater diagnostic accuracy might be achieved by combining structural and functional neuroimaging data within the same SVM, an integrative approach that was successfully applied to an investigation of mild cognitive impairment (Fan et al. 2008b).

\section{Supplementary material}

For supplementary material accompanying this paper visit http://dx.doi.org/10.1017/S0033291713000561.

\section{Acknowledgements}

This work was supported by grants from the National Natural Science Foundation of China awarded to both Q.G. and A.M. (no. 81220108013), Q.G. (no. 81030027 and 81227002) and L.L. (no. 30830046 and 81171286). A.F.M. gratefully acknowledges support from the King's College London Centre of Excellence in Medical Engineering, funded by the Wellcome Trust and EPSRC (Engineering and Physical Sciences Research Council) under grant no. WT088641/Z/09/Z. Q.G. also acknowledges the support from his CMB Distinguished Professorship Award (Award no. F510000/G16916411) administered by the Institute of International Education, USA, and the National Key Technologies R\&D Program (Program no.
2012BAI01B03) and Program for Changjiang Scholars and Innovative Research Team in the University (PCSIRT) of China.

\section{Declaration of Interest}

None.

\section{References}

Ashburner J (2007). A fast diffeomorphic image registration algorithm. Neuroimage 38, 95-113.

Blake DD, Weathers FW, Nagy LM, Kaloupek DG, Gusman FD, Charney DS, Keane TM (1995). The development of a Clinician-Administered PTSD Scale. Journal of Traumatic Stress 8, 75-90.

Brammer M (2009). The role of neuroimaging in diagnosis and personalized medicine - current position and likely future directions. Dialogues in Clinical Neuroscience 11, 389-396.

Burges CJC (1998). A tutorial on support vector machines for pattern recognition. Data Mining and Knowledge Discovery 2, 121-167.

Costafreda SG, Chu C, Ashburner J, Fu CH (2009). Prognostic and diagnostic potential of the structural neuroanatomy of depression. PLOS One 4, e6353.

Davatzikos C, Ruparel K, Fan Y, Shen DG, Acharyya M, Loughead JW, Gur RC, Langleben DD (2005). Classifying spatial patterns of brain activity with machine learning methods: application to lie detection. Neuroimage $\mathbf{2 8 ,}$ 663-668.

Ecker C, Rocha-Rego V, Johnston P, Mourao-Miranda J, Marquand A, Daly EM, Brammer MJ, Murphy C, Murphy DG; the MRC AIMS Consortium (2010). Investigating the predictive value of whole-brain structural MR scans in autism: a pattern classification approach. Neuroimage 49, 44-56.

Fan Y, Batmanghelich N, Clark CM, Davatzikos C (2008a). Spatial patterns of brain atrophy in MCI patients, identified via high-dimensional pattern classification, predict subsequent cognitive decline. Neuroimage 39, 1731-1743.

Fan Y, Resnick SM, Wu X, Davatzikos C (2008b). Structural and functional biomarkers of prodromal Alzheimer's disease: a high-dimensional pattern classification study. Neuroimage 41, 277-285.

First MB, Spitzer RL, Gibbon M, Williams JBW, Davies M, Borus J, Howes MJ, Kane J, Pope HG, Rounsaville B (1995). The Structured Clinical Interview for DSM-III-R Personality Disorders (SCID-II). Part II: Multi-site Test-retest Reliability Study. Journal of Personality Disorders 9, 92-104.

Fu CH, Mourao-Miranda J, Costafreda SG, Khanna A, Marquand AF, Williams SC, Brammer MJ (2008). Pattern classification of sad facial processing: toward the development of neurobiological markers in depression. Biological Psychiatry 63, 656-662.

Gong Q, Wu Q, Scarpazza C, Lui S, Jia Z, Marquand A, Huang X, McGuire P, Mechelli A (2011). Prognostic 
prediction of therapeutic response in depression using high-field MR imaging. Neuroimage 55, 1497-1503.

Hastie T, Tibshirani RFJ (2001). The Elements of Statistical Learning: Data Mining, Inference and Prediction. Springer-Verlag: New York.

Holmes A, Wellman CL (2009). Stress-induced prefrontal reorganization and executive dysfunction in rodents. Neuroscience and Biobehavioral Reviews 33, 773-783.

Klöppel S, Stonnington CM, Chu C, Draganski B, Scahill RI, Rohrer JD, Fox NC, Jack Jr. CR, Ashburner J, Frackowiak RS (2008). Automatic classification of MR scans in Alzheimer's disease. Brain 131, 681-689.

Koutsouleris N, Gaser C, Bottlender R, Davatzikos C, Decker P, Jäger M, Schmitt G, Reiser M, Möller HJ, Meisenzahl EM (2009). Use of neuroanatomical pattern classification to identify subjects in at-risk mental states of psychosis and predict disease transition. Archives of General Psychiatry 66, 700-712.

Lanius RA, Frewen PA, Girotti M, Neufeld RW, Stevens TK, Densmore M (2007). Neural correlates of trauma script-imagery in posttraumatic stress disorder with and without comorbid major depression: a functional MRI investigation. Psychiatry Research 155, 45-56.

Lao Z, Shen D, Xue Z, Karacali B, Resnick SM, Davatzikos C (2004). Morphological classification of brains via high-dimensional shape transformations and machine learning methods. Neuroimage 21, 46-57.

Nardo D, Hogberg G, Looi JC, Larsson S, Hallstrom T, Pagani M (2010). Gray matter density in limbic and paralimbic cortices is associated with trauma load and EMDR outcome in PTSD patients. Journal of Psychiatric Research 44, 477-485.

Norman KA, Polyn SM, Detre GJ, Haxby JV (2006). Beyond mind-reading: multi-voxel pattern analysis of fMRI data. Trends in Cognitive Sciences 10, 424-430.

Orrù G, Pettersson-Yeo W, Marquand AF, Sartori G, Mechelli A (2012). Using support vector machine to identify imaging biomarkers of neurological and psychiatric disease: a critical review. Neuroscience and Biobehavioral Reviews 36, 1140-1152.

Pace TW, Heim CM (2011). A short review on the psychoneuroimmunology of posttraumatic stress disorder: from risk factors to medical comorbidities. Brain, Behaviour, and Immunity 25, 6-13.

Papagni SA, Benetti S, Arulanantham S, McCrory E, McGuire P, Mechelli A (2011). Effects of stressful life events on human brain structure: a longitudinal voxel-based morphometry study. Stress 14, 227-232.

Pereira F, Mitchell T, Botvinick M (2009). Machine learning classifiers and fMRI: a tutorial overview. Neuroimage 45 (Suppl. 1), S199-S209.

Regier DA, Narrow WE, Kuhl EA, Kupfer DJ (2009). The conceptual development of DSM-V. Americal Journal of Psychiatry 166, 645-650.

Robinson BL, Shergill SS (2011). Imaging in posttraumatic stress disorder. Current Opinion in Psychiatry 24, 29-33.

Rosen GM, Taylor S (2007). Pseudo-PTSD. Journal of Anxiety Disorders 21, 201-210.

Sartori G, Pellegrini S, Mechelli A (2011). Forensic neurosciences: from basic research to applications and pitfalls. Current Opinion in Neurology 24, 371-377.

Shin LM, Rauch SL, Pitman RK (2006). Amygdala, medial prefrontal cortex, and hippocampal function in PTSD. Annals of the New York Academy of Sciences 1071, 67-79.

Vapnik V (1995). The Nature of Statistical Learning Theory. Springer-Verlag: New York.

Weathers FW, Litz BT, Huska JA, Keane TM (1994). PTSD Checklist - Civilian Version. National Center for PTSD, Behavioural Science Division: Boston.

Yassa MA, Stark CE (2009). A quantitative evaluation of cross-participant registration techniques for MRI studies of the medial temporal lobe. Neuroimage 44, 319-327.

Yehuda R, Flory JD (2007). Differentiating biological correlates of risk, PTSD, and resilience following trauma exposure. Journal of Traumatic Stress 20, 435-447. 